Portland State University

PDXScholar

Electrical and Computer Engineering Faculty

Publications and Presentations

Electrical and Computer Engineering

$12-1-1975$

\title{
Threshold characteristics of multimode laser oscillators
}

Lee W. Casperson

Portland State University

Follow this and additional works at: https://pdxscholar.library.pdx.edu/ece_fac

Part of the Electrical and Computer Engineering Commons

Let us know how access to this document benefits you.

Citation Details

Casperson, L. W. (1975). Threshold characteristics of multimode laser oscillators. Journal of Applied Physics, 46 (12) 5194-5201.

This Article is brought to you for free and open access. It has been accepted for inclusion in Electrical and Computer Engineering Faculty Publications and Presentations by an authorized administrator of PDXScholar. Please contact us if we can make this document more accessible: pdxscholar@pdx.edu. 


\title{
Threshold characteristics of multimode laser oscillators
}

\author{
Lee W. Casperson \\ School of Engineering and Applied Science, University of California, Los Angeles, California 90024 \\ (Received 3 March 1975)

\begin{abstract}
The oscillation characteristics of a multimode laser are considered in detail, and for the first time saturation by all of the cavity modes is included. Analytical expressions are obtained for the mode It is found that with increasing excitation the spontaneous emission spectrum is gradually resolved into a set of narrow discrete modes. Slightly above threshold the number of modes reduces in a homogeneoulsy broadened laser until only a single mode is oscillating strongly. The theoretical results are in agreement with experimental data that have been reported using semiconductor lasers. With inhomogeneous
\end{abstract} \\ amplitudes, linewidths, and over-all spectral characteristics of lasers operating above and below threshold. \\ broadening the spectral width goes through a minimum near threshold.
}

PACS numbers: $42.60 . J, 42.50$

\section{INTRODUCTION}

Most of the basic features of laser oscillation have been understood for many years. At low excitation levels the output radiation is in the form of a continuous narrow-band emission spectrum characteristic of a Gaussian random-noise process. Above threshold the output is in the form of discrete directional amplitudestabilized modes. In ideal homogeneously broadened lasers, for example, it is well known that above threshold single-mode operation is expected. The lowest-loss mode clamps the gain and prevents oscillation in any other mode. ${ }^{1}$ Even in such an ideal system, however, many features of operation below threshold and in the threshold transition region have not been treated quantitatively. Experimentally, one finds a large number of longitudinal modes oscillating in the vicinity of threshold. Only somewhat above threshold is the spectrum reduced to a single monochromatic mode. ${ }^{2}$

The purpose of this work is to develop a general analytic theory for the output properties of laser oscillators. Saturation due to all of the laser, modes is considered, and the results differ from previous singlemode theories. Good agreement is obtained with experimental data regarding laser output intensity and spectral structure. The analysis is particularly relevant for semiconductor lasers, where the relatively high levels of spontaneous emission can make the threshold transition indistinct. For the same reason the results may be important for a detailed understanding of ultraviolet and potential $x$-ray laser systems. Traditionally, the onset of lasing is observed as an abrupt increase in output intensity. It is shown here, however, that laser systems can be contemplated in which output intensity measurements as a function of excitation yield no information about the threshold transition.

In Sec. II a set of coupled rate equations for the mode intensities is reduced to a single transcendental equation. The total power output is discussed in Sec. III, and other spectral details are considered in Sec. IV. Expressions for the oscillation linewidth are derived in Sec. $V$, and inhomogeneous broadening is treated in Sec. VI.

\section{THEORY}

There are various ways that one can proceed in developing a model for laser oscillation. The details de- pend on the specific problem under consideration and the level of rigor that is required. Our objective here is to develop with minimum complexity a model which includes the basic saturation properties of the FabryPerot cavity modes.

If the oscillation linewidth is narrow compared to the homogeneous linewidth, the intensity associated with a particular mode in a Fabry-Perot laser can be written ${ }^{3}$

$$
I_{j}=\Delta \nu I_{0}\left[1-R_{t} R_{r} \exp (\oint g d z)\right]^{-1}
$$

where $R_{l}$ and $R_{r}$ are, respectively, the reflection coefficients of the left and right mirrors, $g$ is the gain coefficient, $\Delta \nu$ is the longitudinal mode spacing, and the integral represents one loop around the cavity. $I_{j}$ is the total intensity incident on the right-hand mirror and $I_{0}$ is the intensity of spontaneously emitted radiation which has made at most one loop through the cavity. Other cavity losses, if any, are assumed to be included in the mirror reflectivities. If the saturated gain is small and independent of position (uniform pumping, low-loss mirrors), Eq. (1) reduces to

$$
I_{j}=\Delta \nu I_{0} /\left(1-R_{l} R_{r}-2 g l\right),
$$

where $l$ is the length of the amplifying laser medium.

For homogeneously broadened lasers, the noise intensity per mode incident on the right-hand mirror is

$$
I_{0}=\left[h \nu N_{2} / A\left(N_{2}-N_{1}\right)\right]\left[1+R_{l} \exp (g l)\right][\exp (g l)-1] \text { 。 }
$$

Here $N_{2}$ and $N_{1}$ are the total population densities of the upper and lower laser levels, and $A$ is the cross-sectional area of the beam. For simplicity we assume that the lower-level population is negligible, and then Eq.

(3) reduces to

$$
I_{0}=(h \nu / A)\left[1+R_{l} \exp (g l)\right][\exp (g l)-1] .
$$

In this commonly occurring limit the result applies also to inhomogeneously broadened lasers, and the mode characteristics of these lasers are considered in Sec. VI. If the gain is small, Eq. (4) is

$$
I_{0}=2 h \nu g l / A \text {. }
$$

Combining Eqs. (2) and (5) we obtain the expression for mode intensity:

$$
I_{j}=\frac{h \nu \Delta \nu}{A} \frac{2 g l}{1-R_{l} R_{r}-2 g l} .
$$

The intensities of all of the laser modes could be de- 
termined from Eq. (6) if the gain $g$ were known. With saturation, however, the gain is a function of the mode intensities, so the solution is not so easy. First an explicit expression for the intensity and frequency dependence of $g$ must be obtained. It has been shown that in a laser medium with nonnegligible Doppler broadening the intensity of radiation at the frequency $\nu_{j}$ is described by $^{4}$

$$
\frac{d I_{j}}{d z}=\frac{k I_{j}}{\pi} \int_{-\infty}^{\infty} \frac{\exp \left(-\epsilon^{2} y^{2}\right)}{\left[1+\left(y-y_{j}\right)^{2}\right]\left\{1+s I_{j} /\left[1+\left(y-y_{j}\right)^{2}\right]\right\}} d y
$$

where $y(\nu)=2\left(\nu-\nu_{0}\right) / \Delta \nu_{h}$ is a normalized frequency, $k$ is a pumping constant, $s$ is a saturation parameter, and the natural damping ratio $\epsilon=\left(\Delta \nu_{h} / \Delta \nu_{D}\right)(\operatorname{In} 2)^{1 / 2}$ measures the relative importance of homogeneous and Doppler broadening. The homogeneous and Doppler linewidths are given respectively by $\Delta \nu_{h}$ and $\Delta \nu_{D}$. The derivation can be generalized in a straightforward fashion to the interaction with a large number of saturating modes, and the result is

$$
\begin{aligned}
\frac{d I_{j}}{d z}= & \frac{k I_{j}}{\pi} \int_{-\infty}^{\infty} \exp \left(-\epsilon^{2} y^{2}\right) \\
& \times\left[\left[1+\left(y-y_{j}\right)^{2}\right]\left(1+\sum_{n} \frac{s I_{n}}{1+\left(y-y_{n}\right)^{2}}\right)\right]^{-1} d y .
\end{aligned}
$$

Since Eq. (8) describes the growth of intensity, it follows that the gain coefficient for the $j$ th mode is

$g=\frac{k}{\pi} \int_{-\infty}^{\infty} \exp \left(-\epsilon^{2} y^{2}\right)\left[\left[1+\left(y-y_{j}\right)^{2}\right]\left(1+\sum_{n} \frac{s I_{n}}{1+\left(y-y_{n}\right)^{2}}\right)\right]^{-1} d y$.

Our initial interest here is in homogeneously broadened laser media. In the homogeneous limit $\epsilon \gg 1 \mathrm{Eq}$. (9) reduces to

$$
g=g_{h}\left[\left(1+y_{j}^{2}\right)\left(1+\sum_{n} \frac{s I_{n}}{1+y_{n}^{2}}\right)\right]^{-1},
$$

where we have introduced the homogeneous line-center unsaturated gain coefficient $g_{h}=k \pi^{-1 / 2} \epsilon^{-1}$. Equations (6) and (10) may be combined yielding

$$
I_{j}=\frac{h \nu \Delta \nu}{A}\left[\left(\frac{1-R_{l} R_{r}}{2 g_{h} l}\right)\left(1+y_{j}^{2}\right)\left(1+\sum_{n} \frac{s I_{n}}{1+y_{n}^{2}}\right)-1\right]^{-1} \text {. }
$$

This can be regarded as a coupled set of equations governing the intensities of all of the laser modes. These equations must be solved simultaneously in order to obtain a meaningful description of the mode behavior near threshold. In previous analytical treatments it has been assumed that only a single cavity mode has gain. The single-mode approximation is usually not valid, and it cannot predict the important spectral changes which occur in the threshold transition regime. One purpose of the present work is to obtain exact solutions to Eq. (11).

Multiplying both sides of Eq. (11) by $s\left(1+y_{j}^{2}\right)^{-1}$ and summing over all modes yields

$$
x=\frac{\operatorname{sh} \nu \Delta \nu}{A} \sum_{j} \frac{1}{1+y_{j}^{2}} \frac{1}{(1+x) r^{-1}\left(1+y_{j}^{2}\right)-1},
$$

where we have introduced the parameter $r$ $=2 g_{h} l\left(1-R_{l} R_{r}\right)^{-1}$, which is the ratio of the round-trip gain to the round-trip cavity loss, and the intensity parameter

$$
x=\sum_{n} \frac{s I_{n}}{1+y_{n}^{2}}
$$

Equation (12) is now a single equation for the parameter $x$. Once $x$ has been determined, all other properties of the laser output follow readily from Eq. (11).

Using previous definitions, the frequency parameter $y_{j}$ in Eq. (12) can be replaced by $2 j \Delta \nu / \Delta \nu_{h}$ yielding

$$
\begin{aligned}
x= & \frac{\operatorname{sh} \nu \Delta \nu}{A} \frac{1}{(1+x) r^{-1}-1} \sum_{j=-\infty}^{\infty} \frac{1}{1+\left(2 j \Delta \nu / \Delta \nu_{h}\right)^{2}} \\
& \times\left(1+\frac{(1+x)\left(2 j \Delta \nu / \Delta \nu_{h}\right)^{2}}{1+x-r}\right)^{-1} \\
= & \frac{\operatorname{sh} \nu \Delta \nu}{A} \frac{1}{(1+x) r^{-1}-1} \sum_{j=-\infty}^{\infty} \frac{1}{1+a^{2} j^{2}} \frac{1}{1+b^{2} j^{2}}
\end{aligned}
$$

where the definition of $a$ and $b$ are obvious. The summation in Eq. (14) can be performed using the theorem from the calculus of residues ${ }^{5}$

$\sum_{n=-\infty}^{\infty} f(n)=-\sum$ residues of $\pi f(z) \cot (\pi z)$ at the poles of $f(z)$,

and the result is

$\sum_{j=-\infty}^{\infty} \frac{1}{1+a^{2} j^{2}} \frac{1}{1+b^{2} j^{2}}=\frac{\pi a \operatorname{coth}(\pi / a)-\pi b \operatorname{coth}(\pi / b)}{a^{2}-b^{2}}$,

where the relationships $\cot (i z)=-i \operatorname{coth} z$ and $\cot (-i z)$ $=i \operatorname{coth} z$ have been used. With the basic definitions of $a$ and $b \mathrm{Eq}$. (14) now reduces to

$$
\begin{aligned}
x= & \frac{\pi \operatorname{sh} \nu \Delta \nu_{h}}{2 A}\left\{\left(\frac{1+x}{1+x-r}\right)^{1 / 2}\right. \\
& \left.\times \operatorname{coth}\left[\frac{\pi \Delta \nu_{h}}{2 \Delta \nu}\left(\frac{1+x-\gamma}{1+x}\right)^{1 / 2}\right]-\operatorname{coth}\left(\frac{\pi \Delta \nu_{h}}{2 \Delta \nu}\right)\right\} .
\end{aligned}
$$

Equation (17) is a transcendental equation yielding the intensity parameter $x$ for an arbitrary homogeneously broadened Fabry-Perot laser. Numerical solutions can be readily obtained. For most practical lasers, however, the mode spacing is much less than the transition linewidth $\left(\Delta \nu \ll \Delta \nu_{h}\right)$, and in this limit Eq. (17) reduces to

$$
x=x_{0}\left[\left(\frac{1+x}{1+x-r}\right)^{1 / 2}-1\right]
$$

where the spontaneous emission input to the laser is measured by the parameter

$$
x_{0}=\pi \operatorname{sh} \nu \Delta v_{h} / 2 A \text {. }
$$

Equation (18) is a cubic equation in $x$ which can be solved explicitly. ${ }^{6}$ Alternatively, the solutions can be obtained by elementary iterations with a hand calculator. As indicated previously, the parameter $x$ can then be used in Eq. (11) to determine the basic features of the laser output. Of particular interest are the total intensity, the shape of the over-all output spectrum, and the oscillation linewidth. These topics are the subjects of the following sections.

Two regimes of the solutions of Eq. (18) are of particular interest. These are when $r$ is less than unity (below threshold) and when $r$ is greater than unity 


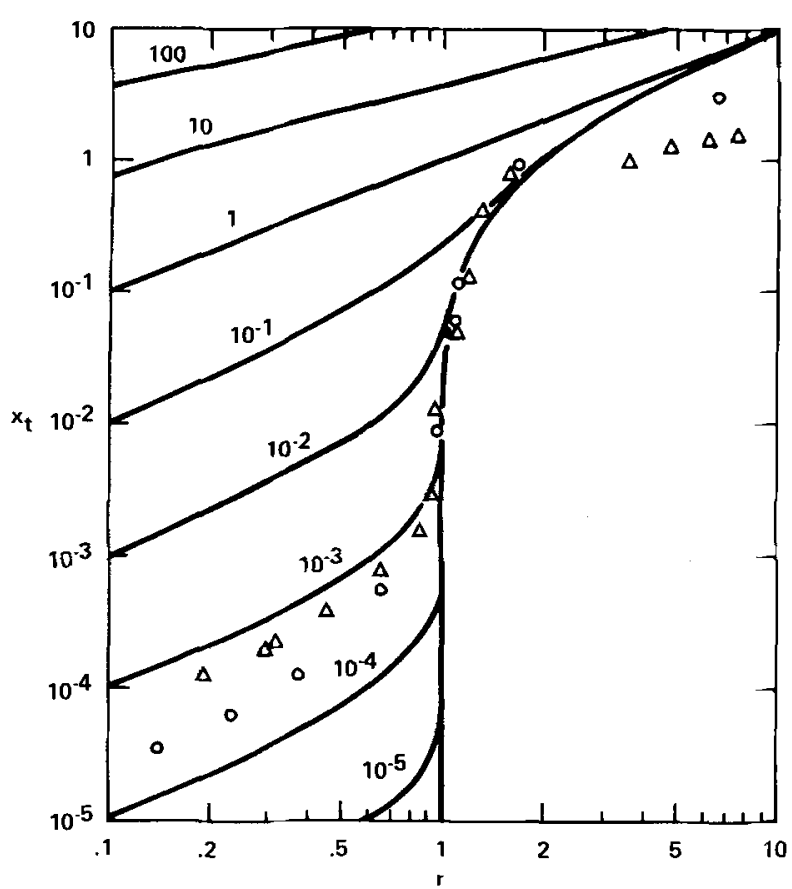

FIG. 1. Total multimode intensity $x_{t}$ in a homogeneously broadened laser as a function of the threshold parameter $r$ for various values of $x_{0}$. The experimental points are from Ref. 8 (triangles) and Ref. 9 (circles).

(above threshold). For the usual case of $x_{0} \ll 1$ we find the two useful limits

$$
\begin{array}{rlrl}
x & =\left[(1-r)^{-1 / 2}-1\right] x_{0}, & & r<1 \\
& =r-1, & r>1 .
\end{array}
$$

These results will be employed in the following discussions.

\section{TOTAL MULTIMODE INTENSITY}

One of the fundamental measurable quantities in a laser oscillator is the total output intensity. Many experiments and applications are sensitive to the intensity and it is important that this quantity be understood theoretically. Multiplying Eq. (11) by $s$ and summing over all modes, we find that the total normalized intensity incident on a mirror is

$x_{t}=\sum_{j} s I_{j}=\frac{\operatorname{sh} \nu \Delta \nu}{A} \frac{1}{(1+x) r^{-1}-1} \sum_{j=-\infty}^{\infty} \frac{1}{1+b^{2} j^{2}}$,

where $b$ is the same as defined previously. The sum can be performed by the residue method yielding

$$
\begin{aligned}
x_{t} & =\frac{\operatorname{sh} \nu \Delta \nu}{A} \frac{1}{(1+x) r^{-1}-1} \frac{\pi}{b} \operatorname{coth}\left(\frac{\pi}{b}\right) \\
& =\frac{r(1+x)^{-1} x_{0}}{\left[1-r(1+x)^{-1}\right]^{1 / 2}} \operatorname{coth}\left[\frac{\pi \Delta \nu_{h}}{2 \Delta \nu}\left(\frac{1+x-r}{1+x}\right)^{1 / 2}\right] .
\end{aligned}
$$

If the mode spacing is much less than the transition linewidth, this reduces to

$$
x_{t}=\frac{r(1+x)^{-1} x_{0}}{\left[1-r(1+x)^{-1}\right] 1 / 2} .
$$

Equation (22) is an explicit expression for the intensity when the parameter $x$ is known, and $x$ is obtained from Eq. (17). For most multimode lasers the approximate forms given in Eqs. (18) and (23) are valid. These results are plotted in Fig. 1 for a wide range of values of $x_{0}$ and $r$. Evidently for small values of $x_{0}$, the intensity increases abruptly by several orders of magnitude when the gain increases past threshold $(r=1)$. However, when $x_{0}$ is large the transition region becomes poorly defined. With $x_{0}=1$, for example, one finds $x_{t}=r$ and there is no transition at all.

Numerous measurements have been reported of the output power of laser oscillators in the vicinity of threshold. Especially interesting for our work are the detailed experimental plots which have been obtained with semiconductor lasers. It is generally believed that the lasing transition in GaAs is primarily homogeneously broadened due to rapid thermalization of the electrons. ${ }^{7}$ Two typical data sets involving electrically ${ }^{8}$ and optically ${ }^{9}$ excited GaAs are reproduced in Fig. 1 fitted roughly to the theoretical curves. Agreement is excellent from well below threshold and through the threshold region. The slight discrepancy above threshold may be due to details of the measurement process, ${ }^{10}$ heating, changes in the waveguide properties, spatial hole burning, or other nonlinear effects. Basically though, the theory we have presented provides a useful description of the laser intensity characteristics over the entire small-signal range.

The data shown in Fig. 1 indicate that for those particular lasers the value of the parameter $x_{0}$ is in the range of $10^{-3}-10^{-4}$. The reasonableness of this value can be readily checked. In terms of the saturation power $P_{s}=A / s$, Eq. (19) is

$$
x_{0}=\pi h \nu \Delta \nu_{h} / 2 P_{s}=\pi h c^{2} \Delta \lambda_{h} / 2 \lambda^{3} P_{s} .
$$

Using typical values for GaAs lasers $\left(\lambda=8700 \AA, \Delta \lambda_{h}\right.$ $=400 \AA$ ) this gives $x_{0}=5.69 \times 10^{-6} P_{s}^{-1}$, where $P_{s}$ is measured in watts. If we take $10 \mathrm{~mW}$ as a reasonable saturation power for oscillation in a single transverse mode, ${ }^{11}$ the result is $x_{0}=5.69 \times 10^{-4}$, which is in the range of the experimental values.

The results also have significant implications for lasers operating at shorter wavelengths. An important feature of Eq. (24) is that the parameter $x_{0}$ is strongly wavelength dependent. Thus, shortening the wavelength by 10 times in the example given previously would increase $x_{0}$ by a factor of $10^{3}$ and the threshold would be completely obscured.

In most conventional lasers, however, the value of $x_{0}$ is much less than unity. In YAG, for instance, with reasonable numbers $\left(\lambda=1.06 \mu, \Delta \lambda_{h}=4.5 \AA, P_{s}=1 \mathrm{~W}\right)^{12}$ Eq. (24) yields $x_{0}=3.5 \times 10^{-8}$. For such small values of $x_{0}$, it is useful to consider limiting expressions for the total intensity. From Eqs. (18) and (23) one obtains

$$
\begin{aligned}
x_{t} & =r x_{0} /(1-r)^{1 / 2}, & & r<1 \\
& =r-1, & & r>1
\end{aligned}
$$

and recourse to the general equations is not required at all.

We have considered so far the intensity characteristics of multimode lasers in which the mode spacing is much less than the over-all linewidth $\Delta v_{h}$. It is also 


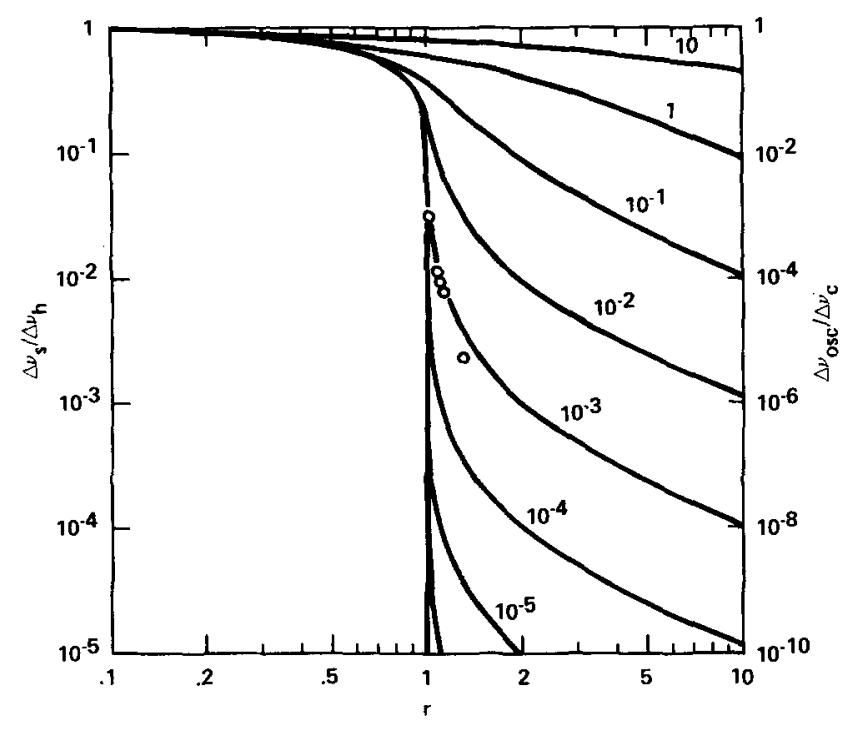

FTG. 2. Width of the Lorentzian mode-spectrum envelope $\Delta v_{s}$ and the individual oscillation modes $\Delta \nu_{\text {osc }}$ in a homogeneously broadened laser as a function of $r$ for various values of $x_{0}$. The experimental data are from Fig. 4 of Ref. 11 and correspond to currents of $740,800,820,840$, and $960 \mathrm{~mA}$.

useful to consider the opposite limit of single-mode oscillation. When the mode spacing is much greater than $\Delta \nu_{h}$, the coth functions in Eqs. (17) and (22) can be replaced by the reciprocals of their arguments yielding

$$
x_{t}=\frac{2 x_{0}}{\pi} \frac{\Delta v}{\Delta \nu_{h}} \frac{r}{1+x_{t}-r}=\frac{x_{0}^{\prime} r}{1+x_{t}-r} .
$$

Thus the intensity for a single mode at line center is governed by a quadratic equation with the solution

$$
x_{t}=\frac{1}{2}\left\{(r-1)+\left[(r-1)^{2}+4 x_{0}^{\prime} r\right]^{1 / 2}\right\} .
$$

For the limits of operation below and above threshold this result reduces to

$$
\begin{aligned}
x_{t} & =r x_{0}^{\prime} /(1-r), & & r<1 \\
& =r-1, & & r>1 .
\end{aligned}
$$

Similar expressions have been obtained in previous single-mode theories. ${ }^{13,14}$ It should be noted that below threshold the single-mode results are distinctly different in form from Eq. (25) and do not correctly describe the intensity behavior of a multimode laser. Also, the single-mode model gives no insight regarding the overall spectral characteristics of the laser output.

\section{OUTPUT SPECTRUM}

We have indicated how the total intensity in a homogeneously broadened laser oscillator can be calculated. Another important characteristic is the oscillator spectrum. Using previous definitions, Eq. (16) can be rewritten in the form

$$
\begin{aligned}
I_{j} & =\frac{h \nu \Delta \nu}{A} \frac{1}{(1+x) r^{-1}\left(1+y_{j}^{2}\right)-1} \\
& =\frac{h \nu \Delta \nu}{A} \frac{r}{1+x-r}\left[1+\left(\frac{1+x}{1+x-r}\right) y_{j}^{2}\right]^{-1} .
\end{aligned}
$$

Thus it is apparent that the envelope of the oscillation mode intensities is always a Lorentzian function, whether the laser is above threshold or not. In real frequency units the full width of the spectrum at half maximum is

$$
\Delta \nu_{s}=\Delta \nu_{h}\left(1-\frac{r}{1+x}\right)^{1 / 2}
$$

The parameter $x$ may be obtained as a solution of Eq. (17). If the mode spacing is much less than the transition linewidth $\left(\Delta \nu \ll \Delta \nu_{h}\right), x$ is obtained from Eq. (18), and $(30)$ reduces to

$$
\Delta \nu_{s}=\Delta v_{h}\left(\frac{x_{0}}{x+x_{0}}\right)
$$

For small values of $x_{0}$, it is useful to consider two limiting regimes corresponding to operation below and above threshold. With Eq. (20) the results are

$$
\begin{aligned}
\Delta \nu_{s} / \Delta \nu_{h} & =(1-r)^{1 / 2}, & & r<1 \\
& =x_{0} /(r-1), & & r>1 .
\end{aligned}
$$

Thus the linewidth below threshold is a function only of the gain and loss, independent of the level of spontaneous emission input.

In Fig. 2 are plots of the spectral width $\Delta \nu_{s}$ from Eqs. (18) and (31) for various values of $x_{0}$. Evidently $\Delta \nu_{s}$ may decrease by several orders of magnitude as the gain increases past threshold. This means that when $r$ is only slightly greater than unity, oscillation is confined mostly to a single longitudinal mode. Precisely this behavior has been widely reported in mode studies with homogeneously broadened lasers. The experimental points in the figure are obtained by fitting Lorentzian envelopes to the mode spectra shown in Fig. 4 of Ref. 11. The data were obtained using a GaAs laser, and apparently the appropriate value of $x_{0}$ is between $10^{-3}$ and $10^{-4}$. This value is in agreement with the GaAs intensity data described previously.

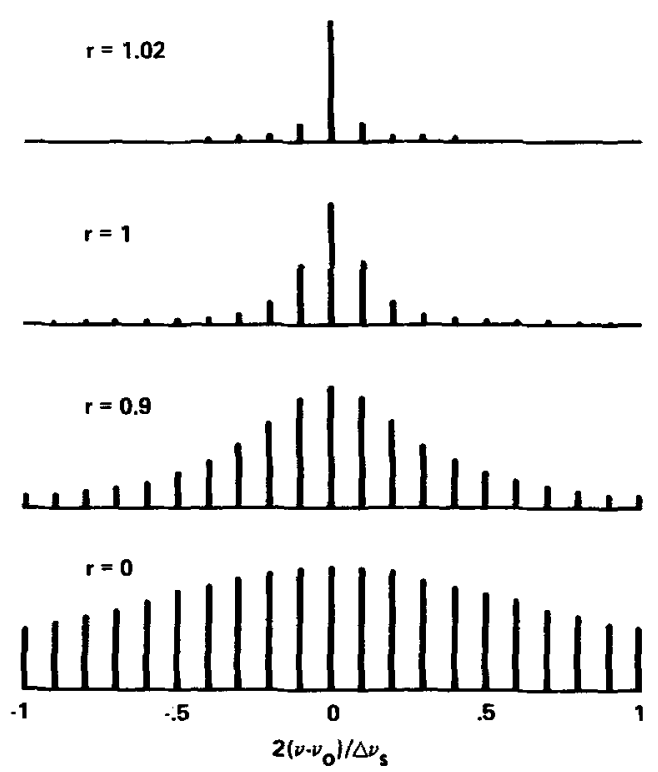

FIG. 3. Longitudinal-mode amplitudes vs normalized frequency $y_{s}=2\left(\nu-\nu_{0}\right) / \Delta \nu_{s}$ for various values of the threshold parameter $r$. In this example the spontaneous emission parameter is $x_{0}$ $=10^{-3}$ and the mode spacing is $\frac{1}{20} \Delta \nu_{h}$. 
Some specific theoretical longitudinal mode spectra are shown in Fig. 3. The mode amplitudes are plotted versus the normalized frequency $y_{s}=2\left(\nu-\nu_{0}\right) / \Delta \nu_{s}$ for various values of the threshold parameter $r$. In this example the spontaneous emission parameter is $x_{0}$ $=10^{-3}$, the mode spacing is $\frac{1}{20} \Delta \nu_{h}$, and the normalization is such that the center mode amplitude is constant. Clearly shown is the rapid line narrowing that occurs near threshold, and the transition is still more abrupt for smaller values of $x_{0}$.

The theoretical expressions derived here are in good agreement with threshold spectral data obtained using semiconductor lasers. Far above threshold, however, a discrepancy begins to occur. The theory predicts continued narrowing (or continued single-mode operation), while in practice the spectral envelope begins to rebroaden. Typically, oscillation of additional modes begins to occur when the gain exceeds losses by a factor of about $2-4(r=2$ to 4$) .^{7-9,11,15}$ Several possible explanations have been proposed including inhomogeneity in the material, spatial hole burning, ${ }^{7,11}$ or some additional nonlinearity. A detailed discussion of this problem has been given. ${ }^{16}$ In practice high-power single-mode output would be best achieved by using laser amplifiers after an oscillator which is operating close to threshold.

We have only considered oscillation in a single tranverse mode. The fundamental tranverse mode is usually desirable for practical applications, and most lasers are designed for this type of operation. In a few situations, however, oscillation in multiple transverse modes is either useful or unavoidable, and a more general theoretical model can be developed. It is necessary first to specify the kind of spatial overlap between the modes. We mention briefly the simplest limit of rapid spatial relaxation. In this limit the transverse modes all compete for the same population inversion and the mathematics is relatively straightforward.

With rapid spatial relaxation the principal difference between the various transverse modes involves the cavity loss and hence the threshold parameter $r$. From Eq. (18) the $x$ parameter corresponding to the firstorder transverse mode is governed by

$$
x_{1}=x_{0}\left[\left(\frac{1+x_{\mathrm{trams}}}{1+x_{\mathrm{trans}}-r_{1}}\right)^{1 / 2}-1\right] \text {, }
$$

where $r_{1}$ is the loss for this mode, and $x_{\operatorname{tran} s}$ is a sum over all transverse modes according to

$$
x_{\text {tr ans }}=x_{1}+x_{2}+x_{3}+\cdots \text {. }
$$

Summing Eq. (33) yields

$$
x_{\mathrm{tr} \text { ans } s}=x_{0} \sum_{n}\left[\left(\frac{1+x_{\mathrm{trans}}}{1+x_{\mathrm{tran}}-r_{n}}\right)^{1 / 2}-1\right] .
$$

This is a single equation which can be solved for $x_{\text {trans }}$ if the $r_{n}$ values of the various transverse modes are known. For stripe geometry lasers the mode-dependent losses can be calculated. ${ }^{17}$ The intensity in any particular mode then follows from Eq. (11) using $x-x_{\text {trans }}$ and the value of $r$ appropriate to the transverse mode order. The total intensity of the longitudinal modes in a transverse mode set follows from Eq. (23), and the spectral width of a mode set is given by Eq. (30).

\section{OSCILLATION LINEWIDTH}

The purpose of this section is to derive expressions for the linewidth of the individual cavity modes. The starting point follows from a straightforward analysis of a Fabry-Perot interferometer containing an amplifying medium. When the lines are narrow compared to the mode spacing, the line shape is approximately Lor entzian with a full width at half-maximum given by

$$
\begin{aligned}
\Delta \nu_{\mathrm{osc}}= & \Delta \nu\left[1-R_{l}^{1 / 2} R_{r}^{1 / 2} \exp \left(\oint \frac{1}{2} g d z\right)\right] \\
& \times\left\{\pi\left[R_{l}^{1 / 2} R_{r}^{1 / 2} \exp \left(\oint \frac{1}{2} g d z\right)\right]^{1 / 2}\right\}^{-1} \\
= & \Delta \nu_{c}\left[\exp \left(\oint \frac{1}{2} g d z\right)\right]^{-1}\left\{1-R_{l}^{1 / 2} R_{r}^{1 / 2}\left[\exp \left(\oint \frac{1}{2} g d z\right)-1\right]\right. \\
& \left.\times\left(1-R_{l}^{1 / 2} R_{r}^{1 / 2}\right)^{-1}\right\}
\end{aligned}
$$

where we have defined the empty-cavity linewidth by

$$
\Delta \nu_{c}=\Delta \nu\left(1-R_{t}^{1 / 2} R_{r}^{1 / 2}\right) / \pi\left(R_{l}^{1 / 2} R_{r}^{1 / 2}\right)^{1 / 2}
$$

If the saturated gain is small and independent of position and if the mirror losses are small, Eq. (36) reduces to

$$
\Delta \nu_{\mathrm{osc}}=\Delta \nu_{\mathrm{c}}\left(1-\frac{g l}{1-R_{l}^{1 / 2} R_{r}^{1 / 2}}\right) \approx \Delta \nu_{c}\left(1-\frac{2 g l}{1-R_{l} R_{r}}\right) \text {. }
$$

With Eq. (10) and the definition of $r$, this is

$$
\Delta \nu_{\text {as } c}=\Delta \nu_{c}\left(1-\frac{r}{(1+x)\left(1+y_{j}^{2}\right)}\right) \text {. }
$$

Equation (39) is an explicit expression for the linewidth when the parameter $x$ is known, and $x$ is given by Eq. (18). Clearly, the narrowest modes are those near line center $\left(y_{j} \approx 0\right)$, which are also the most intense. The width of the line-center modes is

$$
\Delta \nu_{\mathrm{osc}}=\Delta \nu_{c}\left(1-\frac{r}{1+x}\right)
$$

This can be put in an alternative form using Eq. (18) and the result is

$$
\Delta \nu_{\mathrm{osc}}=\Delta \nu_{c}\left(\frac{x_{0}}{x+x_{0}}\right)^{2}
$$

For small values of $x_{0}$ it is again useful to consider the limits corresponding to operation below and above threshold. With Eq. (20) the results are

$$
\begin{aligned}
\Delta \nu_{\mathrm{os} d} / \Delta \nu_{\mathrm{c}} & =1-r, & & r<1 \\
& =\left[x_{0} /(r-1)\right]^{2}, & & r>1 .
\end{aligned}
$$

Equation (41) has the same form as Eq. (31) except that the factor in parentheses in $\mathrm{Eq} .(41)$ is squared. It follows that the oscillation linewidth of the central modes is proportional to the square of the over-all spectral width, independent of the level of pumping. In Fig. 2 is a plot of the oscillation linewidth from Eqs. (18) and (41) for various values of $x_{0}$. Like the spectral width, the oscillation linewidth decreases drastically above threshold.

It is also conventional to express the linewidth of a mode in terms of the total power output from that mode. The output power of the $j$ th mode is related to the internal intensity $I_{j}$ by

$$
P_{j}=\left[\left(1-R_{1}\right)+\left(1-R_{r}\right)\right] A I_{j} \approx 2\left(1-R_{t}^{1 / 2} R_{r}^{1 / 2}\right) A I_{j} .
$$




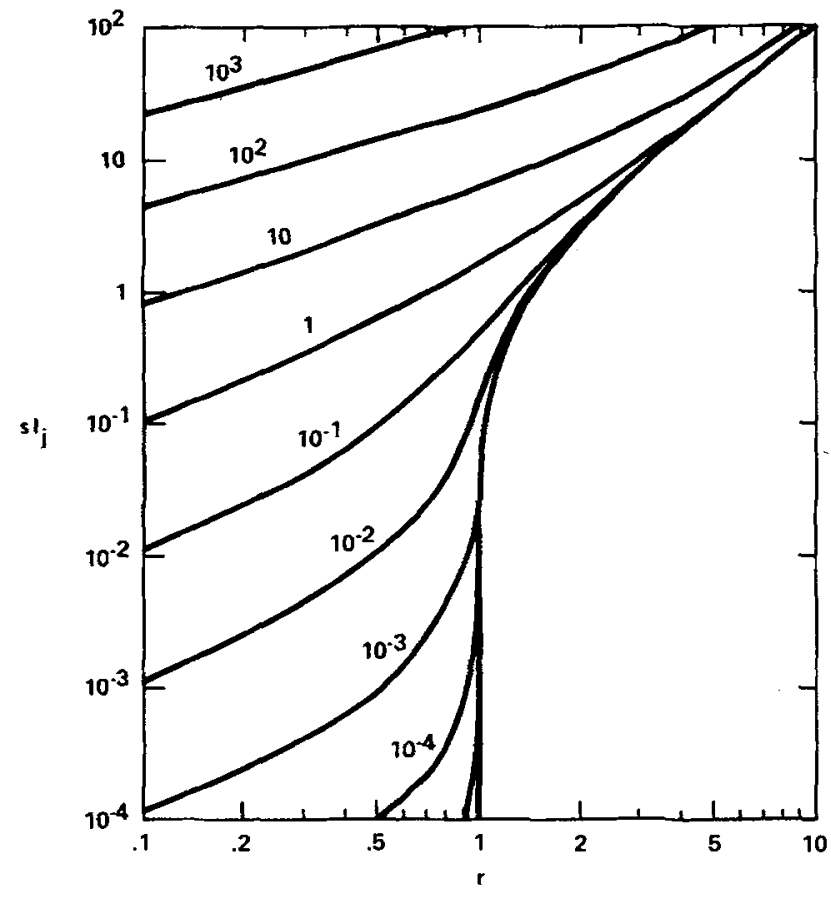

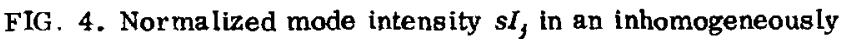
broadened laser as a function of $r$ for various values of $x_{0}^{\prime}$. The mode spacing is la rge compared to $\Delta v_{h}$.

Then with Eqs. (11) and (37) the output power is

$$
P_{j}=\frac{2 \pi h \nu \Delta \nu_{c}}{r^{-1}(1+x)\left(1+y_{j}^{2}\right)-1}
$$

Using Eq. (39), this is

$$
P_{f}=2 \pi h \nu \Delta \nu_{c}\left(\Delta \nu_{c} / \Delta \nu_{a s_{c}}-1\right)
$$

or

$$
\Delta \nu_{\infty \mathrm{c}}=2 \pi h \nu(\Delta \nu)^{2} /\left(P_{j}+2 \pi h \nu \Delta \nu_{c}\right) .
$$

This is a new explicit relationship between the oscillation linewidth and output power, and it is valid for all levels of pumping. Far below threshold the output power is small and we have $\Delta \nu_{\text {osc }} \approx \Delta \nu_{c}$. Above threshold Eq. (46) reduces to the well-known result ${ }^{18}$

$$
\Delta \nu_{a s c}=2 \pi h \nu\left(\Delta \nu_{c}\right)^{2} / P_{j}
$$

\section{INHOMOGENEOUS BROADENING}

The discussion so far has been restricted to the limit of homogeneous broadening, $\Delta \nu_{h} \gg \Delta \nu_{D}$. Similar calculations can be performed when inhomogeneous broadening is dominant. In one limit the various modes interact with different classes of atoms, and the Gaussian function can be removed from the integral in Eq. (9) yielding

$$
g=\frac{k \exp \left(-\epsilon^{2} y_{j}^{2}\right)}{\pi} \int_{-\infty}^{\infty} \frac{d z}{1+s I_{j}+z^{2}}
$$

This can be written

$$
g=g_{1}\left(1+s I_{j}\right)^{-1 / 2}
$$

where $g_{i}=k \exp \left(-\epsilon^{2} y_{j}^{2}\right)$ is the inhomogeneous gain at the laser frequency.

With Eqs. (6) and (49) it follows that the normalized intensity is governed by

$$
\begin{aligned}
s I_{j} & =\frac{h \nu \Delta \nu}{P_{s}}\left[\left(\frac{1-R_{l} R_{r}}{2 g_{i} l}\right)\left(1+s I_{j}\right)^{1 / 2}-1\right]^{-1} \\
& =\frac{x_{0}^{\prime}}{r^{-1}\left(1+s I_{j}\right)^{1 / 2}-1} .
\end{aligned}
$$

This is a cubic equation for the intensity, and the solutions are plotted in Fig. 4 for various values of the parameter $x_{0}^{\prime}$. When $x_{0}^{\prime}$ is small, useful limiting forms of $\mathrm{Eq} .(50)$ are

$$
\begin{aligned}
s I_{f} & =x_{0}^{\prime} r /(1-r), & & r<1 \\
& =r^{2}-1, & & r>1 .
\end{aligned}
$$

As an example, reasonable values for a small $6328-\AA$ helium-neon laser would be $\Delta \nu=7.5 \times 10^{8} \mathrm{~Hz}, P_{s}=10^{-4}$ $\mathrm{W}$ yielding $x_{0}^{\prime}=2.4 \times 10^{-6}$. Thus from Fig. 4 the threshold transition for a particular mode is again highly abrupt.

The oscillation linewidth of a cavity mode can be found by combining Eqs. (38) and (49), and the result is

$$
\Delta \nu_{\mathrm{osc}}=\Delta \nu_{c}\left[1-r\left(1+s I_{f}\right)^{-1 / 2}\right] \text {, }
$$

where the threshold parameter is defined by $r$ $=2 g_{i} l\left(1-R_{l} R_{r}\right)^{-1}$. Equation (52) gives the linewidth explicitly after $s I_{j}$ is found from Eq. (50). The linewidth is plotted in Fig. 5 for various values of $x_{0}^{\prime}$. When $x_{0}^{\prime}$ is small, useful asymptotic formulas are

$$
\begin{aligned}
\Delta \nu_{\cos } \Delta \nu_{c} & =1-r, & & r<1 \\
& =x_{0}^{\prime} /\left(r^{2}-1\right), & & r>1 .
\end{aligned}
$$

It also follows from Eqs. (37), (43), and (50) that the total power output from the $j$ th mode is

$$
P_{j}=2 \pi h \nu \Delta \nu_{c} /\left(r^{-1}\left(1+s I_{j}\right)^{1 / 2}-1\right) \text {. }
$$

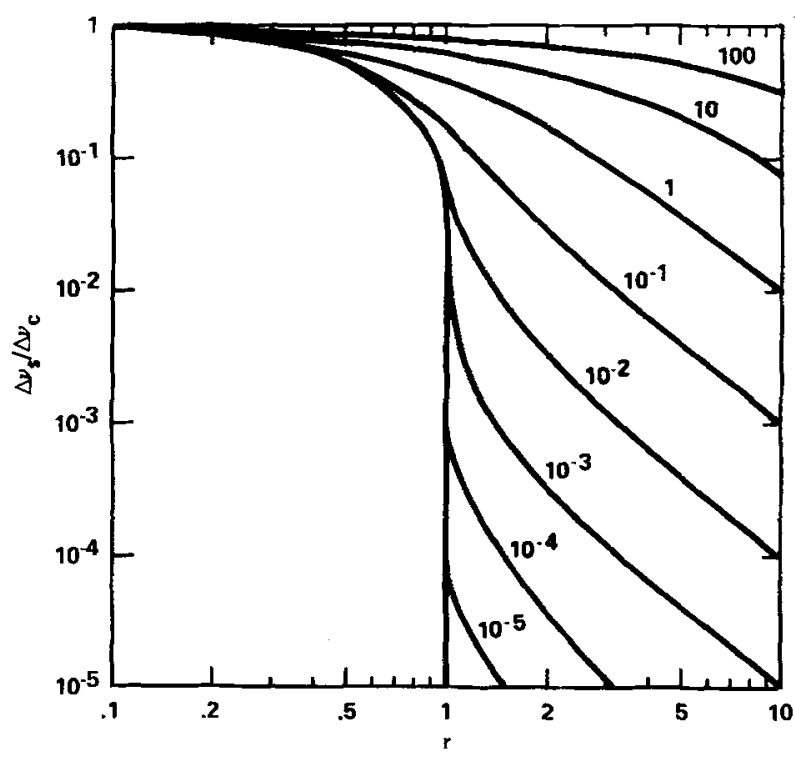

FIG. 5. Oscillation linewidth $\Delta \nu_{\text {osc }}$ in an inhomogeneously broadened laser as a function of $r$ for various values of $x_{0}^{\prime}$. 


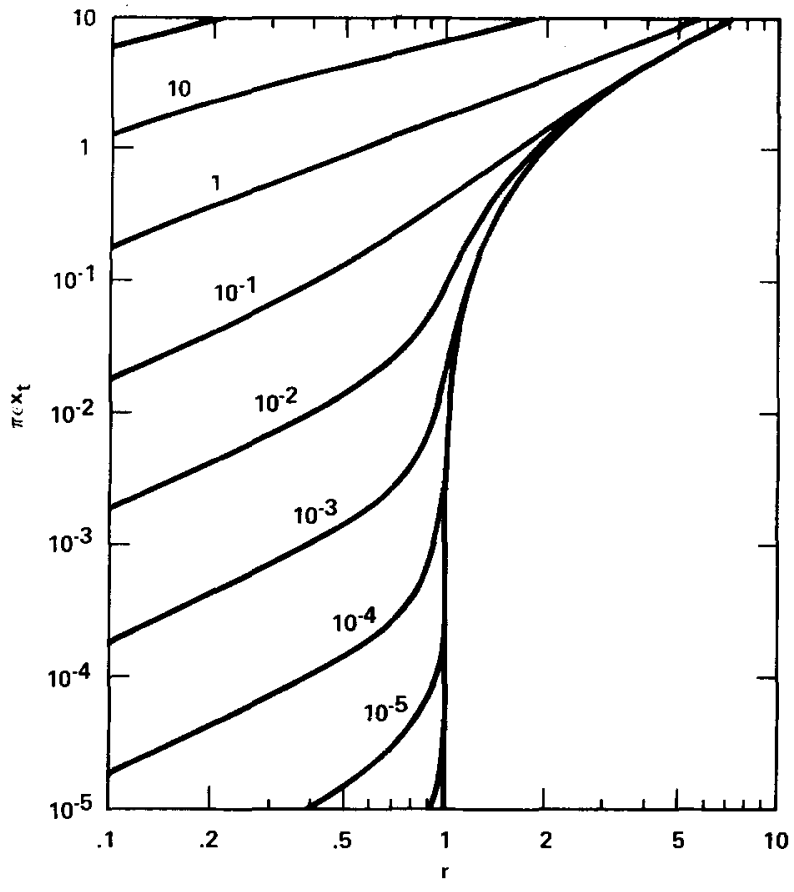

FIG. 6. Normalized multimode intensity $\pi \in x_{t}$ in an inhomogeneously broadened laser as a function of $r$ for various values of $x_{0}$. The mode spacing is small compared to $\Delta v_{h}$.

Therefore, with Eq. (52) the linewidth can be expressed in terms of the mode power according to

$$
\Delta \nu_{\text {os } c}=2 \pi h \nu\left(\Delta \nu_{c}\right)^{2} /\left(P_{j}+2 \pi h \nu \Delta \nu_{c}\right)
$$

which is the same as Eq. (46) governing the linewidth of a homogeneously broadened laser.

The other limit of interest in an inhomogeneously broadened laser occurs when the modes are closely spaced compared to the homogeneous linewidth. Then the mode summation in Eq. (9) can be replaced by an integral, and the gain is

$$
g=\frac{k}{\pi} \int_{-\infty}^{\infty} \frac{\exp \left(-\epsilon^{2} y^{2}\right) d y}{1+\left(y-y_{j}\right)^{2}}\left(1+\int_{-\infty}^{\infty} \frac{s I\left(y_{n}\right) d y_{n}}{1+\left(y-y_{n}\right)^{2}}\right)^{-1}
$$

When the intensity distribution is uniform over a homogeneous linewidth, $I\left(y_{n}\right)$ can be removed from the denominator integral in (56) and the result simplifies to

$$
g=k \exp \left(-\epsilon^{2} y_{j}^{2}\right) /\left[1+\pi s I\left(y_{j}\right)\right]
$$

With Eq. (6) the intensity distribution is

$$
I\left(y_{j}\right)=\frac{\Delta \nu_{h}}{2} I\left(\nu_{j}\right)=\frac{h \nu \Delta \nu_{h}}{2 A} \frac{1}{r^{-1} \exp \left(\epsilon^{2} y_{j}^{2}\right)\left[1+\pi s I\left(y_{j}\right)\right]-1} .
$$

This is a quadratic equation with the solution

$$
\begin{aligned}
s I\left(y_{j}\right)= & \left(\left[r \exp \left(-\epsilon^{2} y_{j}^{2}\right)-1\right]+\left\{\left[r \exp \left(-\epsilon^{2} y_{j}^{2}\right)-1\right]^{2}\right.\right. \\
& \left.\left.+4 x_{0} r \exp \left(-\epsilon^{2} y^{2}\right)\right\}^{1 / 2}\right)(2 \pi)^{-1},
\end{aligned}
$$

where $x_{0}$ is given by Eq. (19). The parameter $r$ is now the ratio of the line-center gain to the loss. The total intensity is obtained by integrating $\mathrm{Eq}$. (59) over all frequencies. With the integration variable $z=\epsilon d y$, the final result can be written

$$
\begin{aligned}
\pi \in x_{t}= & \int_{0}^{\infty}\left(\left[r \exp \left(-z^{2}\right)-1\right]+\left\{\left[r \exp \left(-z^{2}\right)-1\right]^{2}\right.\right. \\
& \left.\left.+4 x_{0} r \exp \left(-z^{2}\right)\right\}^{1 / 2}\right) d z .
\end{aligned}
$$

Equation (60) is plotted in Fig. 6 for various values of $x_{0}$. Evidently, the threshold characteristics are similar to those shown in the previous figures. It also follows from Eq. (60) that for fixed values of $r$ and $x_{0}$ the total intensity is proportional to $\epsilon^{-1}$. For small values of $x_{0}$ these results simplify somewhat in the regions above and below threshold. When $r$ is greater than unity, the upper limit of integration is $(\ln r)^{1 / 2}$ and we obtain

$$
\begin{aligned}
\pi \in x_{t} & =2 x_{0} \int_{0}^{\infty} d z\left[r^{-1} \exp \left(z^{2}\right)-1\right]^{-1}, \quad r<1 \\
& =\pi^{1 / 2} r \operatorname{erf}(\ln r)^{1 / 2}-2(\ln r)^{1 / 2}, \quad r>1 .
\end{aligned}
$$

Equations (60) and (61) would apply, for example, in long 6328- $\AA$ helium-neon lasers, when the mode spacing becomes less than the homogeneous linewidth. Under typical operating conditions the collision-broadened homogeneous linewidth of a helium-neon laser is about $150 \mathrm{MHz}$, so the corresponding cavity length is roughly $L=c\left(2 \Delta \nu_{h}\right)^{-1}=1 \mathrm{~m}$. The parameter $x_{0}$ can be found from Eq. (24).

The spectral width can also be calculated. From Eq. (59) the intensity at line center is

$$
s I(0)=\left\{r-1+\left[(r-1)^{2}+4 x_{0} r\right]^{1 / 2}\right\}(2 \pi)^{-1} .
$$

In terms of this intensity one finds after some algebra that the full spectral width is given by

$$
\Delta \nu_{s}=\Delta v_{D}\left(\ln \frac{2 r\left[\pi s I(0)+2 x_{0}\right]}{[\pi s I(0)]^{2}+2 \pi s I(0)}\right)^{1 / 2}(\ln 2)^{-1 / 2} .
$$

This expression is plotted in Fig. 7. The spectral width goes through a sharp minimum near threshold and then rebroadens back to the Doppler width. For small values of $x_{0}$ the limiting forms of Eq. (63) are

$$
\begin{aligned}
\Delta \nu_{s} & =\Delta \nu_{D}[\ln (2-r)]^{1 / 2}(\ln 2)^{-1 / 2}, & & r<1 \\
& =\Delta \nu_{D}\{\ln [2 r /(r+1)]\}^{1 / 2}(\ln 2)^{-1 / 2}, & & r>1 .
\end{aligned}
$$

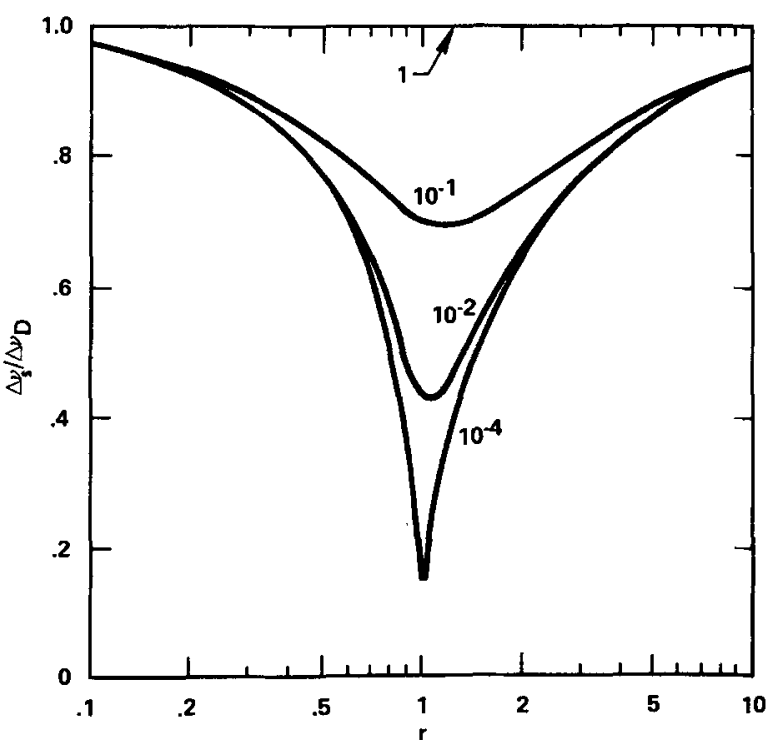

FIG. 7. Width of the mode-spectrum envelope $\Delta \nu_{s}$ in an inhomogeneously broadened laser as a function of $r$ for various values of $x_{0}$. 
We have treated here all of the important limiting regions of laser oscillation. The same general methods also apply in cases of mixed broadening or when substantial spectral variations occur on a scale comparable to $\Delta v_{h}$, but then the analysis becomes more involved. In any event the examples given here include the majority of practical laser oscillators.

\section{CONCLUSION}

A rigorous understanding of the output characteristics of laser oscillators is essential for many practical applications. In this paper we have presented a new formalism which takes into account saturation by the cavity modes. Specific aspects of laser oscillation which have been considered in detail include the total multimode output power, the over-all spectral characteristics, and the linewidth of the individual cavity modes. The predicted behavior in the threshold region is different from that anticipated using previous theories, and the analytical expressions are in agreement with published experimental data. The results are of interest from a theoretical point of view, but in addition they should lead to an improved understanding of existing and proposed laser systems, especially those having substantial spontaneous emission inputs.

${ }^{1}$ T.H. Maiman, Phys. Rev. 123, 1145 (1961).

${ }^{2}$ See, for example, W.V. Smith and P.P. Sorokin, The Laser
(McGraw-Hill, New York, 1966), Sec, 3-3.

${ }^{3}$ L.W. Casperson, Appl. Opt. 14, 300 (1975).

${ }^{4}$ E. I. Gordon, A.D. White, and J.D. Rigden, Symposium on Optical Masers (Polytechnic Institute of Brooklyn, 1963), p. 309.

${ }^{5}$ p. M. Morse and H. Feshbach, Methods of Theoretical Physics (McGraw-Hill, New York, 1953), p. 414.

${ }^{6} \mathrm{M}$. Abramowitz and I.A. Stegun, Handbook of Mathematical Functions, National Bureau of Standards Applied Mathematics Series No. 55 (U.S. GPO, Washington, D.C., 1970), p. 17.

${ }^{7}$ I. A. Poluectov, Yu. M. Popov, and N.N. Shuikin, Proceedings of the Ninth International Conference on the Physics of Semiconductors, edited by S. M. Ryvkin (Nauka, Leningrad, 1968), Vol. 1, p. 613.

${ }^{8}$ W. E. Engeler and M. Garfinkel, J. Appl. Phys. 34, 2746 (1963).

${ }^{9}$ N. G. Basov, A.Z. Grasyuk, V.F. Effimkov, and V.A. Katulin, Sov. Phys. -Solid State 9, 65 (1967).

${ }^{10} \mathrm{For}$ a strict comparison it would be essential, for example, that the detector receive only spontaneous radiation which is emitted into the fundamental mode.

${ }^{11}$ S. Iida, K. Takata, and Y. Unno, IEEE J. Quantum Electron. QE-9, 361 (1973).

${ }^{12}$ T. Kushida, Phys. Rev. 185, 500 (1969).

${ }^{13} \mathrm{H}$. Haug, Phys. Rev. 184, 338 (1969).

${ }^{14}$ A. E. Siegman, An Introduction to Lasers and Masers McGraw-Hill, New York, 1971), Sec. 10-7.

${ }^{15}$ T. L. Paoli, J.E. Ripper, and T.H. Zachos, IEEE J. Quantum Electron. QE-5, 271 (1969).

${ }^{16}$ P.G. Eliseev and N. N. Shuikin, Sov. J. Quantum Electron. 3, 181 (1973).

${ }^{17} \mathrm{Y}$. Suematsu and M. Yamada, IEEE J. Quantum Electron. QE-9, 305 (1973).

${ }^{18}$ A. L. Schawlow and C.H. Townes, Phys, Rev. 112, 1940 (1958). 